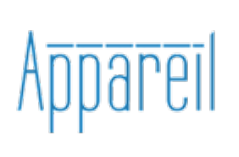

Appareil

$13 \mid 2014$

Trauma et création

\title{
38 témoins de Lucas Belvaux : la schize du témoin
}

Jean-Louis Déotte

\section{(2) OpenEdition}

Journals

Édition électronique

URL : http://journals.openedition.org/appareil/2082

DOI : 10.4000/appareil.2082

ISSN : 2101-0714

Éditeur

MSH Paris Nord

Référence électronique

Jean-Louis Déotte, « 38 témoins de Lucas Belvaux : la schize du témoin », Appareil [En ligne], 13 | 2014, mis en ligne le 03 novembre 2014, consulté le 30 juillet 2020. URL : http://journals.openedition.org/ appareil/2082 ; DOI : https://doi.org/10.4000/appareil.2082

Ce document a été généré automatiquement le 30 juillet 2020.

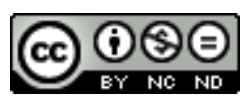

Appareil est mis à disposition selon les termes de la Licence Creative Commons Attribution - Pas d'Utilisation Commerciale - Pas de Modification 4.0 International. 


\title{
38 témoins de Lucas Belvaux : la schize du témoin
}

\author{
Jean-Louis Déotte
}

\section{NOTE DE L'ÉDITEUR}

Nous remercions Lucas Delvaux ne nous avoir fourni gracieusement ces photogrammes tirés de son film 38 témoins (2012).

1 Pourquoi la ville du Havre fut-elle détruite entre le 5 et le 12 septembre 1944 par un bombardement britannique d'une rare intensité alors qu'une bonne partie du pays était libérée, que le général commandant la place n'était pas un jusqu'au-boutiste et ses troupes, peu aguerries? On nous dit qu'il fallait pouvoir utiliser un port pour ravitailler les troupes alliées et que Le Havre était le plus proche. Mais l'exemple de Cherbourg l'avait montré en juin : un port détruit doit être débarrassé de ses épaves, ce qui prend du temps. Dans le même ordre d'idée : pourquoi les autorités civiles françaises (maire et préfet) ne donnèrent-elles jamais l'ordre d'évacuation de la ville de Caen, faisant subir à la population un terrible calvaire ? On nous dit : pour empêcher les pillages allemands... 1

Quel fut l'état existentiel d'un survivant-témoin de la destruction de ces villes?

3 Aujourd'hui, dans ces villes reconstruites, seul un événement dramatique et absurde peut jeter un pont vers ces destructions gratuites. 


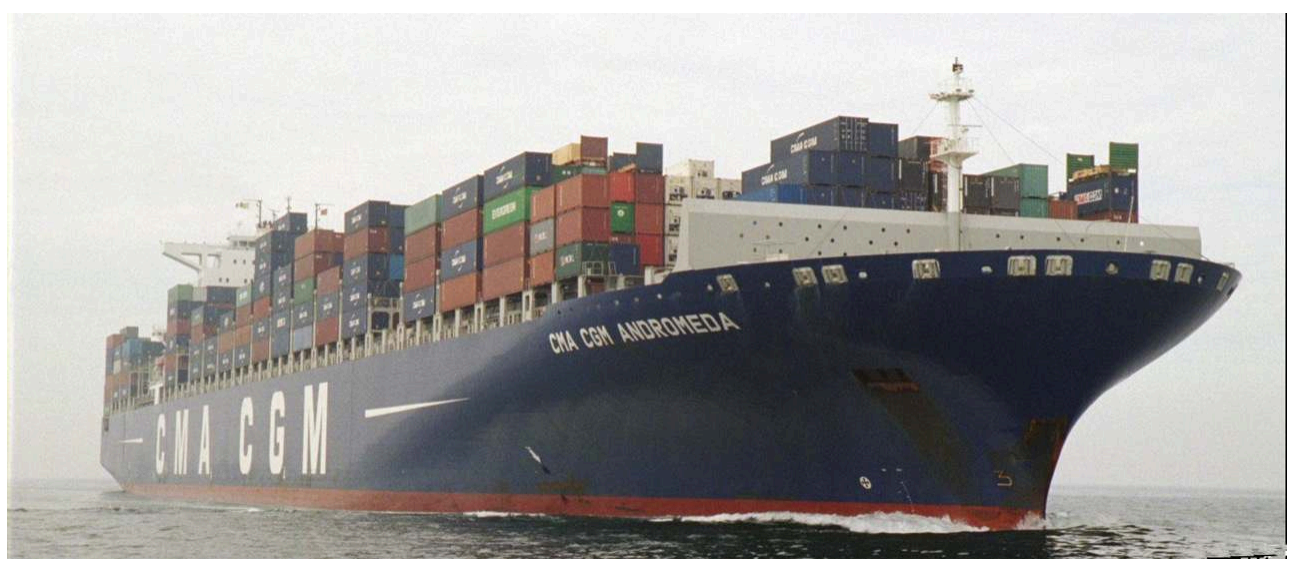

La proue noire d'un énorme porte-conteneurs s'impose dès les premières images du film 38 témoins ${ }^{2}$, c'est celle de l'Andromède, que Pierre, pilote du Havre, va prendre en charge. Dans la mythologie grecque, Androméda est une princesse éthiopienne. Fille du roi Céphée, elle a été victime de l'orgueil de sa mère Cassiopée. Exposée nue sur un rocher pour y être dévorée par un monstre marin, elle est sauvée de justesse par Persée dont elle deviendra l'épouse. Son nom pourrait signifier: "celle qui dirige les

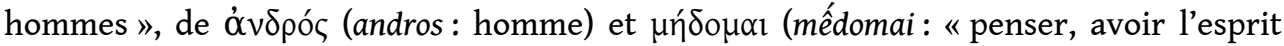
plein de »). Ce mythe sera transposé au Moyen Âge en combat de saint Georges avec le dragon.

5 Il est pourtant difficile d'identifier le pilote Pierre (Y. Attal) qui dirige l'Andromède vers son havre, avec Persée, à moins de considérer que le monstre marin de la mythologie a pris la figure des Érinyes: Mégère (Méүaı / Mégaira, «la Haine»), Tisiphone

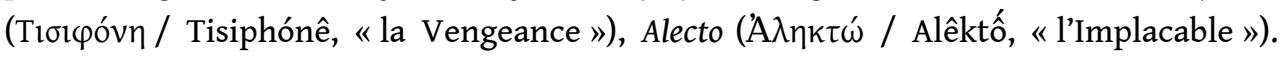
C'est pourtant la menace que brandira le procureur de la République. Le procureur reprend ici le rôle du chœur dans la tragédie classique, celui du rappel du bon sens civique, des valeurs de la Cité : il est inutile de rappeler ce que nous savons tous, les choses étant ce qu'elles sont, le peuple est atteint de cécité et d'amnésie, à moins de vouloir attiser la haine, la vengeance implacable contre un quartier entier. Certes le pilote aura pu, contre tous, restaurer son sens de la responsabilité, confirmer en apparence son autonomie morale au sens de la loi éthique chez Kant, mais ce sera au prix fort de celui par qui le scandale advient. Les média sont nos puissances chtoniennes que tous craignent, même les puissants. Et il n'est pas sûr qu'il suffise pour un coupable devant une assemblée populaire (commissions de vérité et de réconciliation) de demander pardon aux proches de ses victimes. Le film de Belvaux nous place en deçà de la problématique de la culpabilité collective et du pardon, c'est ce que les critiques n'ont pas perçu, pas plus que la provocatrice Marcella Iacub.

6 Ce qui devrait alerter notre intelligence des ères de la cosmétique, et de leurs différends, c'est que pour la post-moderne Ariane, pour Louise l'épouse du pilote, il n'y a pas plus d'énigme (le Minotaure) que de mystère chrétien. À la différence des labyrinthes classiques, la postmodernité industrielle offre à l'humanité des labyrinthes mouvants, ceux, par exemple des engins de levage du port. Le monstre n'est pas au centre du labyrinthe, il est dans la programmation du déplacement des engins qui rend imprévisible le chemin à suivre. Le risque pour Louise peut surgir de partout. Ariane n'a plus de fil à dévider dans un univers sans repères symboliques. Car c'est bien cette absence qui devient notre lot commun après une destruction de masse. 38 témoins est un film sur les survivants d'une destruction psychosociale de masse, ce qui renforce le 
contraste avec la beauté de la ville reconstruite par Perret et la qualité d'une esthétisation de l'industrie.

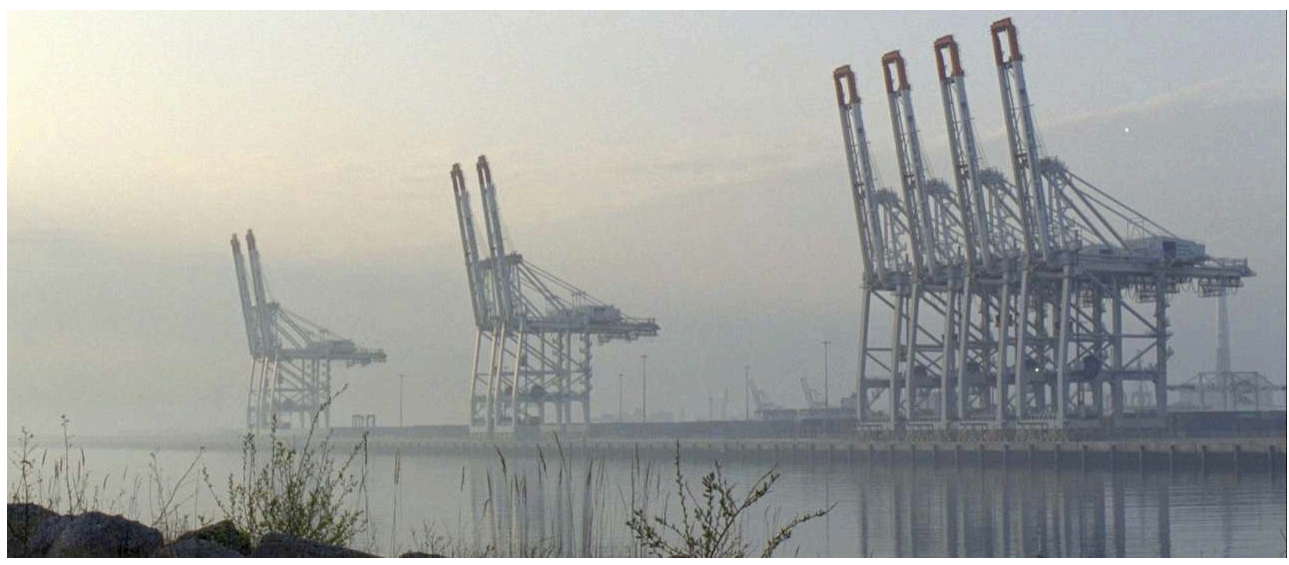

7 Là où Didier Decoin dont le livre Est-ce ainsi que les femmes meurent ? (2010), qui a inspiré Belvaux, en bon auteur omniscient de l'ancien temps, nous dévoile les faits et gestes de la victime, Kitty Genovese, et du monstre qui l'assassina et la viola, dorénavant, ignorant tout, nous serons tous dans la situation du témoin. Nous appartenons à l'âge du témoignage, ce qui fait aussi de nous des contemporains.

\section{Mais est-on jamais le témoin d'un événement ?}

8 C'est la reproduction du cri lors de la reconstitution policière qui va amener Louise à prendre la décision de quitter le pilote. Le cri reconstitué, théâtral, est plus terrible que le cri initial, authentique, parce qu'il a un sens, alors que le premier donna lieu à de multiples interprétations plausibles, comme le rappelle le procureur. Cela a une conséquence philosophique importante: la reproduction ou représentation, en amplifiant l'événement, c'est-à-dire en l'inscrivant, en l'imitant, est plus proche de la vérité que ce qui a été vécu. La représentation purifie l'événement en écartant l'inessentiel.

Mais les motivations de Louise peuvent être différentes : on peut penser aussi que si elle décide de tout quitter, son quartier et le pilote, c'est qu'elle a compris que la vie ne sera plus possible avec ces voisins-là, avec cet amant-là.

Ou encore, qu'à leur place, elle n'aurait pas agi autrement, rejoignant ainsi la cohorte de la supposée lâcheté collective.

C'est qu'à la différence des 38 témoins, Louise connaît dorénavant le sens exact du cri. C'est une femme qui a été assassinée là et non pas la querelle d'un couple de pochards. Elle est alors le témoin idéalisé par les média : celui qui sait à quoi il a assisté. Mais c'est une pure fiction, puisque Louise était absente. Le témoin idéal serait celui qui connait le sens de l'événement quand il a lieu. C'est ce qu'on nous demande d'être avec le « travail de mémoire». Cela débouche sur l'illusion que l'histoire a un sens et qu'il y a un tribunal qui, à la fin, dira le sens de l'histoire: «l'histoire jugera » déclare-t-on à la suite de Hegel.

Mais en fait, on ne peut témoigner du vécu immédiat d'un événement, car s'il s'agit d'un événement par définition inouï, et non pas d'une quelconque péripétie, quand il a eu lieu, personne n'était capable d'en prendre conscience dans sa radicale altérité. C'est la raison pour laquelle l'accueil d'un véritable événement ne peut être que négatif, ce 
n'est donc pas un accueil, mais un refus, un NON ! On ne dit pas spontanément OUI ! à l'événement sauf si l'habitude nous a préparés à le recevoir. L'hospitalité quant à l'étranger, cet extra-terrestre, est donc problématique, relève Derrida ${ }^{3}$. On ne peut accueillir que ce que l'on connaît un tant soit peu, le presque familier. Malgré toutes leurs bonnes et mauvaises raisons, les voisins auditifs ne pouvaient pas devenir de bons témoins. Didier Decoin s'évertue à imaginer que si les voisins de Kitty Genovese, assassinée dans le Queens à New York en mars 1964 en sortant d'un bar par une froide nuit d'hiver, n'ont pas alerté immédiatement la police, c'est qu'ils se vengeaient ainsi d'une femme qui vivait avec une autre femme. Il écarte ainsi le dilemme du psychosociologue Stanley Milgram : « sommes-nous capables en cas de danger avéré de nous porter assistance ou sommes-nous des grains de poussière les uns pour les autres? ». Milgram est connu pour ses travaux sur l'acceptation de l'obéissance la plus absurde quand la personne qui donne les ordres a l'aura de la science objective la plus légitime. Les psychologues Bibb Latané de l'Université de Columbia et John Darley de l'Université de New York conduisirent des expériences du même type que celles de Milgram sur le syndrome Kitty Genovese ou bystander effect (le bystander est une personne qui assiste à un événement sans s'y impliquer). Les chercheurs en conclurent que "quand un seul témoin est présent dans une situation d'urgence, il porte la responsabilité de devoir l'assumer; mais si d'autres sont présents, la charge de la responsabilité se diffuse $»^{4}$. Mais à cette heure de la nuit, on ne peut pas supposer un collectif de témoins... Comme l'écrit Benjamin, si les rêves sont collectifs, les réveils sont solitaires.

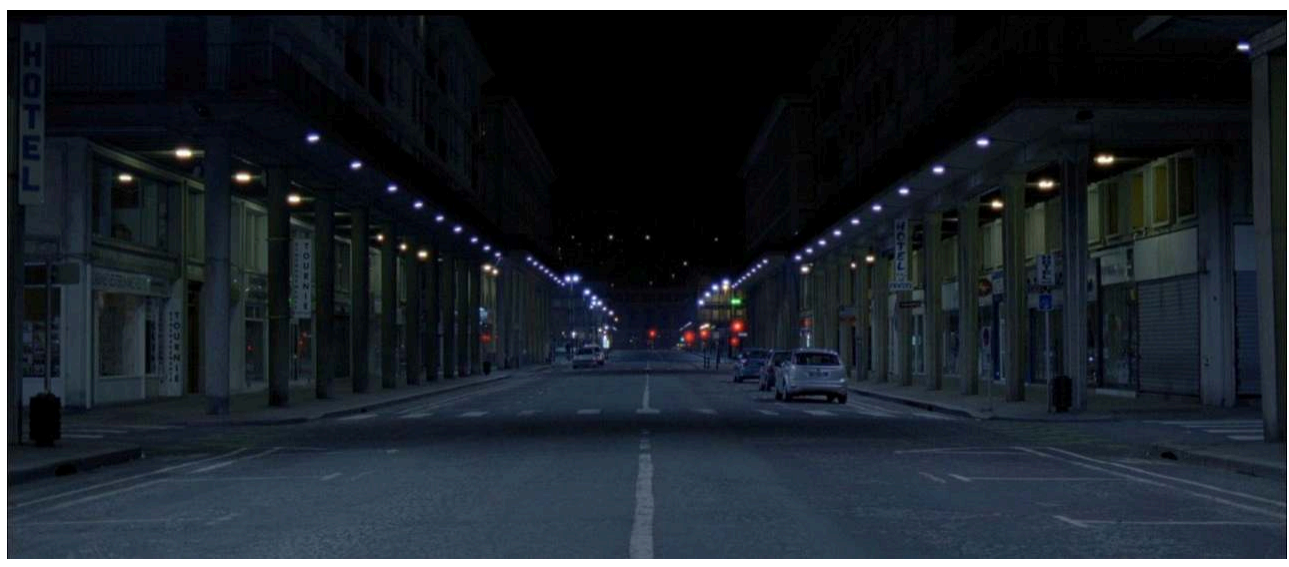

\section{Le cri}

Dans ses deux confessions, l'une à Louise, l'autre à la journaliste locale, le pilote tente de caractériser ce que fut son vécu cette nuit-là. Il décrit le cri dans sa quintessence. Ses qualités sont d'être: "épouvantable pour un humain", "inouï », "on ne peut imaginer, dit-il, qu'il provienne d'un humain». Le cri «traverse toutes les parois", psychologiques, physiologiques et matérielles. «Fracasse le cerveau comme le ferait une balle ", "vide le corps de son sang ", « l'envahit d'adrénaline ». Le cri « écrase tous les membres». Il n'y a donc plus de surface d'inscription subjective. L'événement " détruit donc toute possibilité de témoignage " et " pourtant on ne pourra l'oublier ", "pas plus qu'on ne pourrait l'imaginer", on ne peut l'identifier conceptuellement. Celui qui l'a perçu et qui accepte de décrire son état, le pilote, caractérise son état depuis cette destruction physico-physiologico-psychique : «il n'existe plus, mais il est 
le seul à le savoir », il subsiste « entre la vie et la mort », comme « un fantôme » et cela ne cessera jamais, quoi qu'il fasse d'important.

sens aigu de la responsabilité, qu'il a toujours la capacité d'obéir à des règlements et d'observer des savoir-faire techniques? Tournons-nous vers les distinctions établies par Kant dans : Réponse à la question : qu'est-ce que les Lumières ?, un texte qui s'éloigne de la problématique du transcendantal, c'est-à-dire de la philosophie de la connaissance, pour interroger l'histoire comme émancipation de l'humanité. À le suivre, l'entendement ne cesse pas de connaître (d'où le respect continu des normes techniques indispensables à l'exercice du métier), mais la raison, celle qui nous oblige après coup à interroger le sens des ordres reçus, est invalidée. Bref, le cri ou le pur événement disjoignent l'entendement et la raison pratique et condamnent le «témoin» à la minorité qui est la situation d'une humanité réduite à l'enfance, dominée, hétéronome, privée des Lumières. Hétéronome au sens de : recevant la loi d'ailleurs, s'opposant à l'autonomie de celui qui agit en fonction d'une loi que l'humanité majeure se donne à elle-même. En demandant justice, le pilote espère retrouver l'état de majorité, la capacité, qui n'est légitime que dans l'après-coup selon Kant, d'interroger les finalités de l'action réglementaire, afin, dans le cadre d'un échange épistolaire avec des pairs, d'en interroger le sens. Peut-on imaginer un cyborg interrogeant la finalité de son action?

18 Que serait aujourd'hui la représentation possible d'une humanité se détournant de la capacité d'interroger les finalités de son action? Une "post-humanité » dont les capacités sensorielles seraient décuplées par de nouveaux appareillages, capables d'engendrer des sons et des images inouïs, tout cela piloté par des capacités de calcul et de mémoire gigantesques. On reconnait la fantasmagorie "post-humaine », celle d'une humanité augmentée (Sloterdijk $\mathrm{k}^{7}$ ), d'un corps immortel par autoréparation, autoengendrement, autoproduction (Jean-Michel Besnier ${ }^{8}$, Dominique Babin ${ }^{9}$, Dominique Lecourt $\left.{ }^{10}\right)$. Mais ces fables sont d'une très grande naïveté, on fait l'économie de la 
politique et de l'histoire : celle des destructions de masse, dans des guerres classiques ou asymétriques, celle des terrorismes d'État (politique de disparition, etc.). Bref, on n'imagine pas qu'il puisse y avoir, d'une manière irréductible à la constitution des sociétés, des différends cosmétiques donnant lieu à des guerres civiles immémoriales, comme l'actuelle confrontation entre Chiites et Sunnites.

Le cri, par définition, c'est la phôné, c'est l'animalité selon Aristote, c'est l'affect inarticulé, c'est le hors langage de l'enfant. Pour les classiques, comme Rancière aujourd'hui, il y a toujours la possibilité d'une articulation de l'affect, du tort, il n'y a pas de reste. Entre l'entendement et la raison, au sens de Kant, on peut toujours selon lui construire des ponts. C'est un optimisme habermassien. Pour Lyotard au contraire, entre le cri et le logos, il y a un abîme infranchissable, un différend. Donc un reste irrelevable. Par bien des côtés, en particulier si l'on aborde la question des affects qui eurent lieu et pourtant ne passent pas, comme dans le cas d'Emma analysé par Freud, quelque chose est définitivement enkysté qui pourtant continue d'agir dans le présent du patient.

Mais ici, ce cri, après avoir été perçu dans sa répétition, oblige le pilote à partir, à quitter sa femme, son métier, sa ville pour rejoindre un bateau où son histoire ne sera pas connue et où il deviendra un pur anonyme.

On est donc bien obligé d'affirmer l'existence d'événements qui génèrent un tort absolu pour des victimes en quelque sorte collatérales dont la minorité sera éternelle parce qu'elle ne porte pas sur le courage d'avoir pu témoigner ou pas, d'avoir trouvé des témoins ou pas, mais une humanité comme celle de l'enfant Useppe de la Storia d'Elsa Morante $^{11}$ atteint d'épilepsie.

Car il n'y a pas que l'événement d'un cri inhumain qui puisse dissocier l'entendement et la raison : certaines images ont le même pouvoir.

L'humanité est ainsi constitutivement épileptique.

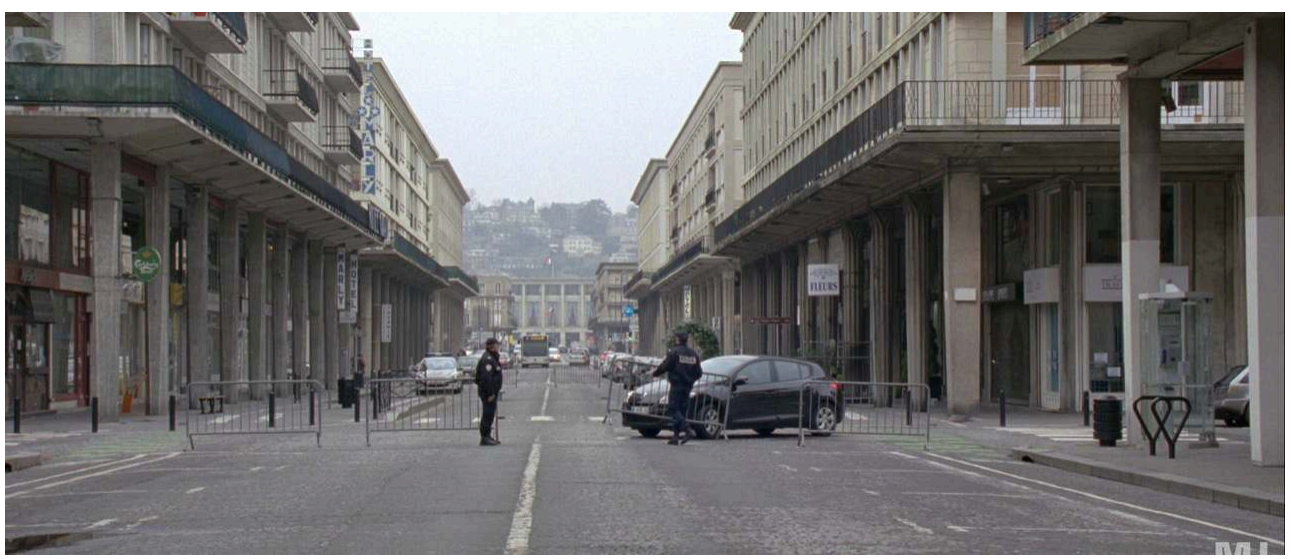

\section{BIBLIOGRAPHIE}

Babin Dominique, Manuel d'usage et d'entretien du post-humain, Paris, Flammarion, 2004. 
Besnier Jean-Michel, Demain les posthumains. Le futur a-t-il encore besoin de nous?, Paris, Fayard, 2010.

Decoin Didier, Est-ce ainsi que les femmes meurent?, Paris, Grasset \& Fasquelle, 2009.

Derrida Jacques, Dire l'événement, est-ce possible ? Séminaire de Montréal par Jacques Derrida, Paris, L'Harmattan, coll. « Esthétiques », 2001.

Hastings Max, Overlord, manque l'année et l'édition

Kant, Que signifie s'orienter dans la pensée ? manque l'année et l'édition

Lecourt Dominique, Humain, posthumain, Paris, Presses universitaires de France, 2003.

Morante Elsa, La Storia, Paris, Gallimard, [1974], 2004.

Sloterdijk Peter, Règles pour le parc humain, suivi de : La domestication de l'être, trad. Olivier Mannoni, Paris, Fayard/Mille et une nuits, 2011.

\section{Films}

Belvaux Lucas, 38 Témoins, 2012.

Romero George A., La nuit des morts vivants, 1968.

\section{NOTES}

1. Pour ce qui est de la destruction de Caen, l'historien britannique Max Hastings, dans son livre Overlord, décrit le bombardement comme «une des attaques aériennes les plus futiles de la guerre » car les Allemands étaient positionnés au nord de la ville et non dans la ville même.

2. 38 Témoins, film de Lucas Belvaux, 2012, adapté du roman de Didier Decoin, Est-ce ainsi que les femmes meurent?, Paris, Grasset \& Fasquelle, 2009.

3. Jacques Derrida, Dire l'événement, est-ce possible ? Séminaire de Montréal par Jacques Derrida, Paris, L'Harmattan, coll. « Esthétiques », 2001.

4. Didier Decoin, op. cit., p. 183.

5. Au sens de Romero : La nuit des morts vivants, film de 1968.

6. Il est donc bien en deçà de la différenciation corporelle native entre la droite et la gauche que suppose Kant dans Que signifie s'orienter dans la pensée?.

7. Peter Sloterdijk, Règles pour le parc humain, suivi de: La domestication de l'être, trad. Olivier Mannoni, Paris, Fayard/Mille et une nuits, 2011.

8. Jean-Michel Besnier, Demain les posthumains. Le futur a t-il encore besoin de nous?, Paris, Fayard, 2010.

9. Dominique Babin, Manuel d'usage et d'entretien du post-humain, Paris, Flammarion, 2004.

10. Dominique Lecourt, Humain, post-humain, Paris, Presses universitaires de France, 2003.

11. Elsa Morante, La Storia, Paris, Gallimard, [1974], 2004.. 


\section{RÉSUMÉS}

L'article ne porte pas sur la lâcheté des témoins, ce que tout le monde a écrit, mais sur l'idéologie du post-humanisme, d'une "humanité augmentée ». Car les auteurs de cette fantasmagorie ne peuvent décrire qu'une humanité obéissante, mineure, réduite à n'exercer que son entendement selon le Kant de « Réponse à une question : qu'est-ce que les Lumières? ». Je fais l'hypothèse que la situation traumatique vécue par la population de ce quartier havrais ne lui permet pas d'accéder à la majorité, c'est-à-dire à la capacité de discuter sur une place publique des finalités de la raison. La question pourrait être élargie : «pourquoi les bourreaux de crime de masse ne parlent-ils pas?»

\section{INDEX}

Mots-clés : banalité du mal, post-humanisme, droits des robots 